\title{
SoccerMate: A Personal Soccer Attribute Profiler using Wearables
}

\author{
H M Sajjad Hossain, Md Abdullah Al Hafiz Khan, Nirmalya Roy \\ Department of Information Systems, University of Maryland Baltimore County \\ riaj.sajjad@umbc.edu, mdkhan1@umbc.edu,nroy@umbc.edu
}

\begin{abstract}
The use of smartphone and wearable devices in various sporting events is an optimistic opportunity to profile player's physical fitness and physiological health conditioning attributes. Recently a variety of commercial wearables with respect to different sports are available in the market. As these wearables differ for distinctive sports, it becomes a hassle to effectively profile them for multiple sports sessions in day to day practice events. Wrist worn devices like smartwatches are becoming a trend in sports analytics recently and researchers are leveraging them to infer various contexts of the players to improve the quality, tactics, strategy of playing matches against the opponents. Visual observation is the most popular way to track a player's abilities in soccer, but as a player it is not always possible to self-assess your own strengths and weaknesses in a field. In this paper, we propose to exploit the wrist worn devices with built in accelerometer to help represent attributes of technical judgement, tactical awareness and physical aspects of a soccer player. We propose to use deep learning to build our classification model which analyzes different soccer events like in-possession, pass, kick, sprint, run and dribbling. Based on these soccer events, we evaluate the overall ability of a soccer player. Our experiments show that, these wearable technology guided attributes profiling can help a coach or scout to better understand the competence of a player in addition to traditional visual observation.
\end{abstract}

\section{INTRODUCTION}

Recent trends in wearable technologies have opened up a large number of application domains which involve identifying human behavior, activity and physiological contexts. These applications and research are augmenting different sensor modalities to build diverse analytics using machine learning techniques. For example, health and fitness applications like monitoring exercise, inferring sleep patterns, gait analysis etc. (using accelerometer, heart rate sensor) have been investigated intensively in the past. Health analytics help users to understand the limit of their locomotive movements and physiological metrics and how they can improve reach and overcome those limitations. This interpretation of health attributes has also assisted to create analytics in various sporting domains. The use of sensor technology has been adapted in many sports recently and it is facilitating to investigate and model professional performances of players involved. For example, Sensoria [1] a fitness t-shirt that can track acceleration forces and other vitals has been utilized in Formula one racing, Catapult [2] sensor tracks about 100 metrics and is now used in professional NFL and NBA games, Motus baseball [3] sleeve and Zephyr Bioharness [4] Heart analyze players performance in baseball games. A very promising and encouraging news is that Germany national soccer team used wearable technology to profile the players and with the statistics coach Joachim Low made the crucial substitute of Mario Gotze who scored the winner in the world cup final [5]. However all commercially available products are used in professional setting and for different sports there are different devices. As wrist worn devices are getting more and more popular, researchers are exploiting the in built accelerometer for inferring activity context information. Making these devices multi-functional can help to reduce the need for buying other function specific wearable devices. In this paper, we propose to use these multifunctional devices to extract metrics of a soccer player.

Soccer which is also known as Football in different parts of the world, is the most popular known sports in the universe we live in. About 250 million players in over 200 countries and dependencies are engaged in playing soccer with about 3.5 billion fans all over the world. All technical, tactical, physical and psychological attributes are involved in this game. When profiling a soccer player there are many attributes which scouts and coaches look into according to the position the player plays in. Mostly visual observation plays a vital role when judging the performance of a player. Some general attributes for a soccer player includes - pace, shooting, passing, dribbling, defending and physical. These attributes also encompass some low level metrics. These low level metrics compile the high level attributes previously mentioned. For example, for high level attribute passing low level metrics like vision, short and long passing, crossing and free kick need to be calculated. It is evident that these metrics are hard to quantize using just technology. For example, how many passes was completed or free kick was on target or scored needs to be logged separately. In our work, we first try to infer the low level metrics using the wrist worn device and then attempt to define aggregated high level attributes by taking the weighted average. We take the weighted average because some low-level metrics are more important than others based on the position the players are playing. For example, as a playmaking midfielder vision is very important on the other hand as a striker, finishing which involves kicking is more important than other attributes. Using our model anyone can track their soccer attributes without any professional setting or prior training in real life.

\section{RELATED WORKS}

There has been a lot of research on improving sports experience and understand capabilities of players using wearable 
technology. SwimMaster [6] extracts various swim parameters like time spent per lane, speed and the number of strokes using accelerometer sensor in real-time. The authors of [7] proposed a basketball event monitoring system using various body worn sensors which detects human movements like walking, jogging, running and shooting the ball. Rajiv et al. [8] applied recurrent neural network to analyze basketball trajectories. The authors of [9] developed a smart soccer shoe embedded with pressure sensors which detects the interaction with the ball and performs shot analysis based on contact speed and angle. Jaime et al [10] developed a body sensor network to monitor the physical state of each player where each player is considered as a node in the wireless sensor network. The authors of [11] demonstrated a soccer pose estimation system using multiple uncalibrated camera. Sentioscope [12] analyzes soccer videos and tracks players using model field particles. The authors of [13] proposed a model which generates highlight of a soccer game automatically using wearable sensors by detecting events like instep-kick and ball speed.

Researchers are also using soccer game data to analyze the performance of a team by applying machine learning algorithms on various open datasets of professional soccer games [14] [15] [16] [17] [18]. QPass [19] analyzes the passing ability of a soccer player using historical data. [20] proposed a soccer analytics system which uses FIFA soccer video game data and described the characteristics of FC Barcelona's distinguished style in 2012/13 La Liga season. Yasuo et al. [21] proposed a real-time prediction system to come up with tactics to win the game using the data of the first half. A data-driven player ranking model based on passes completed is demonstrated in [22]. A variety of promising commercial devices are also coming into the market through which a player's profile can be projected. Germany national soccer team utilized Adidas miCoach wearable technology to profile their players which helped them to secure the world cup in 2014 [5]. In this paper, we focus on utilizing daily wearable technologies to profile generic attributes of a casual soccer player which can help him improve his skills without relying on professional technologies.

\section{DeEP LEARning Model}

We used deep learning method Restricted Boltzman Machine (RBM) to classify low level soccer metrics. A Restricted Boltzmann Machine is a stochastic neural network meaning there are neuron-like units whose binary activations depend on the neighbors they are connected to and these activations are probabilistic. RBM model is composed of three layers - visible, hidden and bias. Each neuron in visible layer is connected to all the hidden neurons in the hidden layer. The bias unit is connected to all the neurons in both visible and hidden layer. RBM maintains a $H \times V$ weight matrix $W$ where $V$ denotes number of visible neurons and $H$ is the number of hidden neurons. Each $w_{i j}$ represents weight between visible neuron $v$ and hidden neuron $h$. Each connection is also associated with bias weights in each layer, $b_{i}$ for visible layer and $c_{j}$ for hidden layer. RBM in general, works by updating

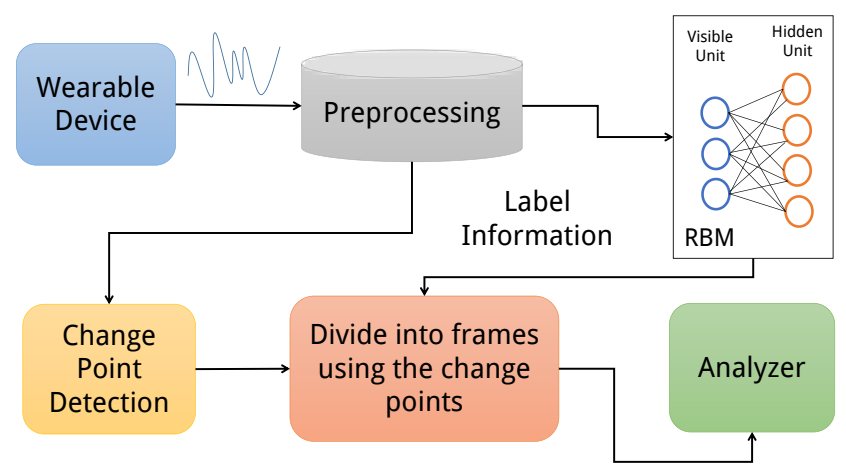

Fig. 1: An architectural overview of SoccerMate model.

the states of some neurons given the states of others using the energy function. The energy function and joint distribution of visible and hidden layer are defined as following

$$
\begin{aligned}
& E(v, h)=-\sum b_{i} v_{i}-\sum c_{j} h_{j}-\sum W_{i} j b_{i} h_{j} \\
& P(v, h)=\frac{1}{Z} \exp \{-E(v, h)\}
\end{aligned}
$$

In eqn $2, Z=\sum_{v} \sum_{h} \exp (-E(v, h))$ is the normalizing constant partition function. In order to maximize this joint distribution, we need to minimize the energy $E$. In our case the accelerometer data follows Gaussian distribution and the visible layer receives continuous real valued data. As a result Gaussian visible neurons have to be introduced in the visible layer. This model uses probability values instead of samples from the Gaussian distribution as input. The energy function for Gaussian RBM is defined as following

$$
E(v, h)=\sum \frac{\left(v_{i}-b_{i}\right)^{2}}{2 \sigma_{i}^{2}}-\left(\sum c_{j} h_{j}+\sum \frac{v_{i}}{\sigma_{i}^{2}} h_{j} w_{i j}\right)
$$

In the above equation $\sigma_{i}$ is the variance of the input data. The activation energy is scaled by the variance which reduces the mean square error between the input and the output that results in better reconstruction. An architectural overview of our model is demonstrated in figure 1.

\section{Change Point Detection}

For providing smoother label to the continuous input, our model detects change points in the dataset and divides a stream of data into frames. Each frame symbolizes a potential event (sprint, pass, kick, dribbling, ball touch) and each frame size is different as the length of the events are different. Finding the change points also helps us to understand the duration of each events. We have multivariate (3-axis) data from the accelerometer and using change point detection we want to estimate differences between data distributions. The authors of [23] [24] proposed a divergence measure using which we can compare whether two independent random vectors are identically distributed. Let us consider two random vectors $X$ and $Y$ their characteristics functions are $\phi_{x}(t)$ and $\phi_{y}(t)$ respectively. According to [24] a divergence measure between these two distributions is defined as following 

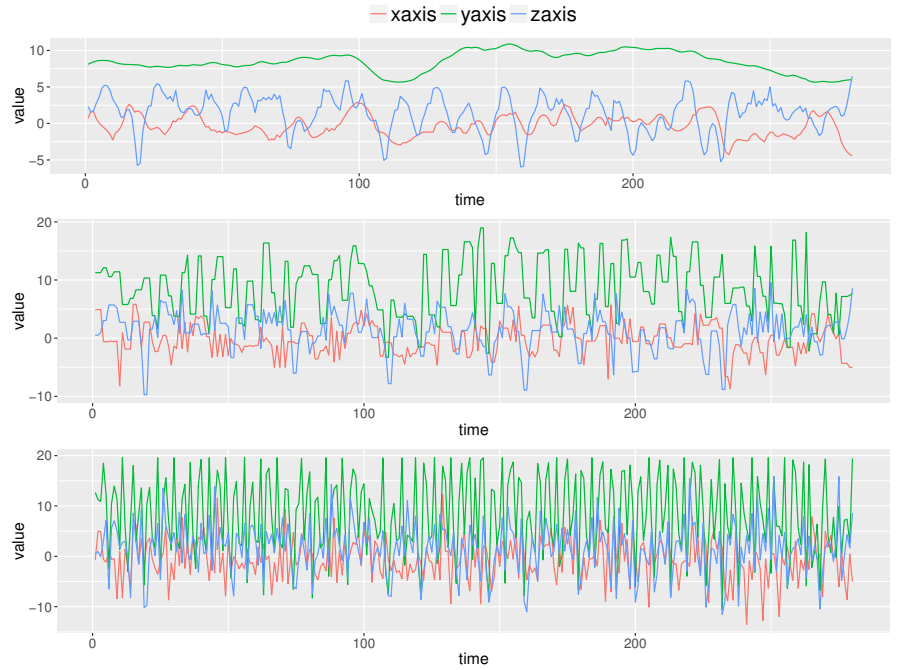

Fig. 2: The figure illustrates our the effect of filtering. The bottom figure is the plot of raw accelerometer data. The data looks like the middle figure after applying median filter. The top figure depicts the signal after applying kalman filter.

$$
\begin{aligned}
& \int\left|\phi_{x}(t)-\phi_{y}(t)\right|^{2} w(t) d t \\
& w(t ; \alpha)=\left(\frac{2 \pi^{d / 2} \Gamma(1-\alpha / 2)}{\alpha 2^{\alpha} \Gamma[(d+\alpha) / 2]}|t|^{d+\alpha}\right)^{-1}
\end{aligned}
$$

In the equation $4, w(t)$ is a positive weight function which is defined according to [25]. We compute multiple change points by computing the divergence measure iteratively to find a single change point. At each iteration a change point is detected and the change points segments the data stream. Consecutive two change points define a frame.

\section{ANALYZER}

From our change point detection module, we create frames of individual soccer events. Using these frames we extract and calculate some statistics to score a player. After getting the label information of the data we calculate different attributes $(A)$ like average speed $\left(S_{\text {avg }}\right)$, sprint speed $\left(S_{\text {sprint }}^{t}\right)$, acceleration $A$, number of passes $(P)$, number of kicks $(K)$, number of dribbles $(R)$, total running distance $(D)$. We exploit the coefficients $(C)$ defined in EA Sports FIFA 2013 as weights in our model for these attributes. According to the position the player is playing the coefficient values change. In Table I position specific coefficient values are shown. We calculate the score of individual player using equation 5 . We normalize the score in the range of 1 to 10 and get the final rating.

$$
W_{\text {score }}=\sum C_{i} A_{j}
$$

\section{PREPRocessing}

Playing soccer involves a lot of random movements for example different hand gestures, charging and grabbing an opponent etc. These random movements impose noise in our

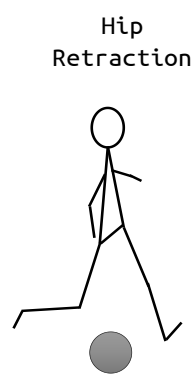

(a)

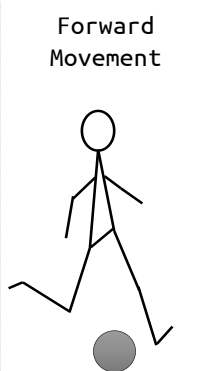

(b)

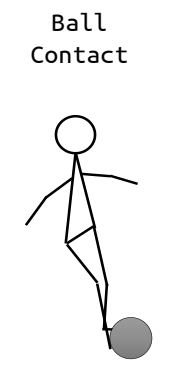

(c)

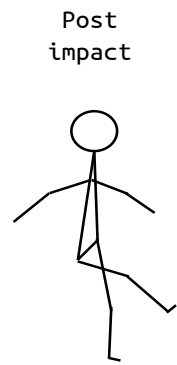

(d)
Fig. 3: The figure demonstrates the biomechanics of the body when passing/kicking the ball. (a) backswing phase of the kicking leg (b) forward movement for kicking (c) ball in contact with the leg (d) post follow through after kicking the ball

dataset, so we apply filters to smooth the data. First we apply a sliding window based moving median filter that removes the noise while preserving the signal pattern. In the second step we apply a Kalman filter. Using the Kalman filter we estimate and minimize the mean squared error by observing the data stream which enabled to produce more prominent patterns in the data. From figure 2 we can see the effect of filtering and the filtered data is much smoother. Another problem we have to deal with is the class imbalance problem. In a soccer game, a player posses the ball for a very small percentage of time. So most of the physical movements are - walking, standing and running. To deal with this imbalance problem, we oversample the minor class instances.

\section{Soccer Events \& Movements}

In this section we discuss the soccer events we considered for our model along with their biomechanics. The performance attributes are same for all the players other than the goalkeeper despite the differences in their position and the importance of these attributes vary in different positions. For example, a player playing as a defender will be judged based on his defending (tackling, marking, blocking etc.) skills or a midfielder with a playmaker role will be judged based on his playmaking abilities (vision, through pass, number of passes, crosses etc.) Our goal is to focus on general activities of all the soccer players. We discuss the general activities in the following

- Passing: This is the most basic and most perceived event while playing soccer. This event lasts no longer than 5 seconds depending on the decision of the player. The goal is to project the ball to another player of the same team using leg. The force is generated from the swing of the leg and longer the pass harder the kick. According to newton's third law of motion - For every action, there is an equal and opposite reaction, the body experiences a backward force for which we see a subtle but distinguishable change in the accelerometer data.

- Kicking: This event is a more intense version of passing where the force is much higher. 
TABLE I: Position specific coefficients of the attributes

\begin{tabular}{|c|c|c|c|c|c|c|c|}
\hline Position & Avg Speed & Sprint & Acceleration & Pass & Kick & Dribbles & Distance \\
\hline Center Back & 0.04 & 0.02 & 0.01 & 0.05 & 0.01 & 0.00 & 0.06 \\
\hline Wing Back & 0.06 & 0.06 & 0.05 & 0.10 & 0.10 & 0.07 & 0.13 \\
\hline Central Midfielder & 0.09 & 0.04 & 0.03 & 0.14 & 0.05 & 0.09 & 0.06 \\
\hline Winger & 0.07 & 0.05 & 0.05 & 0.14 & 0.06 & 0.11 & 0.11 \\
\hline Attacking Midfielder & 0.12 & 0.08 & 0.04 & 0.16 & 0.11 & 0.11 & 0.16 \\
\hline Striker & 0.10 & 0.04 & 0.05 & 0.04 & 0.10 & 0.20 & 0.05 \\
\hline
\end{tabular}

- Walking: When not in ball possession the player roams around in the field.

- Running: A player may run with the ball or without the ball depending on the situation of the game.

- Standing: A player sometimes stands and waits for the ball or keeping the ball while standing and deciding in which direction he should pass the ball.

- Dribbling: There are many kinds of tricks a player can perform to confuse the opposite player and move past him with the ball. We consider two basic dribbling techniques - Cut and Step Over. The best dribblers in the world touch the ball with every step which enables more control over the ball. Cut means a player move past an opponent just by changing direction and more control over the ball results in successful cut dribbling. Step over was one of the signature dribbling move of Brazilian legend Ronaldo where a player circles and moves his feet over the ball.

\section{A. Biomechanics}

The primary goal of SoccerMate is to infer the events where a player interacts with the ball. According to our event list passing and kicking are such events. In figure 3 we demonstrate the biomechanics of passing/kicking event. The backswing of the leg accumulates the force for the kick and the angular velocity of the thigh is low and the velocity of the lower part of the leg is negative due to the backward movement. From figure 3(a) it is also noticeable that the hand on the kicking limb side also starts to experience a backward movement. Initially during the forward motion the hand starts to stretch out further away from the body $3(\mathrm{~b})$. At the point of ball contact $15 \%$ of the kinetic energy due to the swinging leg is transferred to the ball and the arms stretch out further 3(c). After the ball contact in the post impact phase the body reacts to the force applied to the ball 3(d). The post impact phase is very important as this follow through of leg helps the player to protect himself from injury by gradually depleting the kinetic forces generated by the kicking. It is evident from the figure that the hand stretches the most at this phase and the length of the stretch depends on the force of the kick. For a light touch it is possible that the hand movement will not be visible.

\section{EXPERIMENTAL RESULTS}

In this section we evaluate our SoccerMate model based on following three criteria.

- Accuracy: We illustrate the accuracy of our RBM classifier using overall accuracy, precision, recall and Fmeasure.
- Resource Usage: We monitor the network and memory usages of our SoccerMate model in smartphone.

- Energy Consumption: We compute the total energy consumption of SoccerMate deployed in a smartphone

\section{A. Implementation}

We developed an android application using a centralized architecture where the data collected using the wrist worn devices are uploaded in bulk after a session has ended. The data is uploaded in a Microsoft Azure web server. The SoccerMate application invokes an Azure Machine Learning web service which applies the filtering and change point detection algorithm on the session data. The server side computation was done using R-script. We trained our RBM classifier offline using Torch and deployed the trained model in the mobile device. Our RBM model consists of 3 hidden layers with 256 nodes in each layer.

\section{B. Data Collection}

We collected data at a sampling rate of $32 \mathrm{~Hz}$ using two different wrist worn devices - Actigraph GT3X and Empatica Embrace. Both devices have built in accelerometer through which we captured the acceleration along $x, y$ and $z$ axis. We collected data from 6 players who were playing casual soccer in a 5 a side game. Each session provided 1 hour of data and we collected data for 10 sessions. Out of these 6 players, two were playing as forward, two as midfielder and two as defender. Before the start of each session, we asked the participant to put on the wearable on his non-dominant hand. The participants were also asked to provide information about his playing position from three options (Forward, Midfielder and Defender) and his dominant leg (two-footed, right footed, left footed) using the android app. We compare our analytics with one of the commercially available devices - Zepp soccer sensor [26]. The participants put on the Zepp sensor on their dominant leg using a calf sleeve, if two footed then on any one of the leg. The ground truth data was collected using video recording for all of the sessions. Data from four sessions were used as training set and rest of the data were used in the testing phase.

\section{Evaluation}

After filtering the data, we try to find the change points in the dataset which reflect the start, end or a transition of a performed event. Using the classifiers output it is possible to calculate that but in such case change points were detected too frequently which did not help us in doing the player's performance analysis. In figure 4 , change points detected on a window of the dataset is shown. 


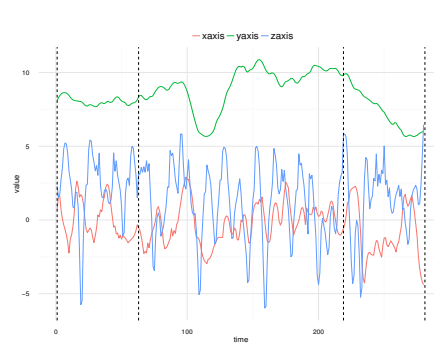

Fig. 4: Dotted lines denote detected change points

Fig. 5: Precision, Recall and F1 score of our SoccerMate model.

1) Accuracy: We received an overall accuracy of $86.54 \%$, however we experienced very high accuracy for walking, running and standing. In figure 5 we plot the Precision, Recall and F-1 score of our model. It is evident that walking, running and standing events were most accurately inferred. For other events we received much lower accuracy. The reason we received low accuracy for pass event is that the biomechanics of passing/kicking is truly visible in ideal cases. In real game, many constraints are involved while passing the ball. For example, if you are playing one touch football or a 1-to-1 pass, most of the time the interaction with the ball will be very delicate and the receiver just changes the direction of the ball without putting any real force to it. Also while you are running with the ball, the ball is already in motion and for a short pass it is not necessary to put a lot of force on the ball. Players also kick the ball in different ways (inside or outside of the boot) which pose more difficulty in finding pattern in the data and ultimately resulted in low accuracy $(\approx 75 \%)$. Kick event exhibits better accuracy $(\approx 82 \%)$ than passing as more force is involved which results in much more intense jerk of the body. We achieved lowest accuracy for dribbling event. As discussed in previous section we intend to infer the cut and step over dribbles. During the cut dribbling the player shifts his body balance abruptly and cut the ball in a different direction. During our experiments we noticed that unless there is a change in the speed of the player, the accelerometer data do not reflect the event clearly. For the step over event, the player circles over his feet over the ball and then accelerates toward a certain direction. The classifier inferred some dribbling instances as walking because in those cases the speed of the player was same before and after he performed the move.

2) Resource Usage: Our smartphone application does not collect data in real time. At the start of each session the participants pair their wearables with their smartphones and start playing. Accelerometer data are logged in wearable device memory while playing and synced with the smartphone application using bluetooth communication protocol after the session has ended. The average data file size is around 5.3 $\mathrm{MB}$ and on average data syncing consumes 53.4 Joules of energy. The memory usage including the synced data file and our trained model is $7 \mathrm{MB}$. The average CPU usage of our application is $6 \%$.

TABLE II: Comparison of performance attributes using Zepp Sensor for each player in one session

\begin{tabular}{|c|c|c|c|c|c|}
\hline Player & Pass & Position & Kick & Sprint & Total Distance $(\mathrm{m})$ \\
\hline Player1 & $23 / 28$ & Defender & $3 / 5$ & $1 / 2$ & $2.8 / 2.7$ \\
\hline Player2 & $36 / 27$ & Midfielder & $4 / 7$ & $3 / 3$ & $3.92 / 4.32$ \\
\hline Player3 & $11 / 4$ & Striker & $1 / 3$ & $1 / 1$ & $1.13 / 1.38$ \\
\hline Player4 & $22 / 16$ & Striker & $2 / 3$ & $2 / 4$ & $2.76 / 3.25$ \\
\hline Player5 & $19 / 14$ & Defender & $0 / 2$ & $3 / 2$ & $1.98 / 2.33$ \\
\hline Player6 & $28 / 29$ & Midfielder & $5 / 9$ & $6 / 5$ & $2.65 / 3.12$ \\
\hline
\end{tabular}

3) Network Usage: As filtering and change point detection algorithm are the most resource hungry steps, we use a centralized architecture where we process the data on the server side to mitigate the energy consumption of the mobile device. After receiving the session data from the wearable, we invoke an Azure ML web service. The web service sends back the filtered data along with change points information. If using $3 \mathrm{G}$ while invoking the web service, uploading session data and downloading returned output requires 160 Joules of energy on average, however if using Wifi the average energy consumption is 61.3 Joules only.

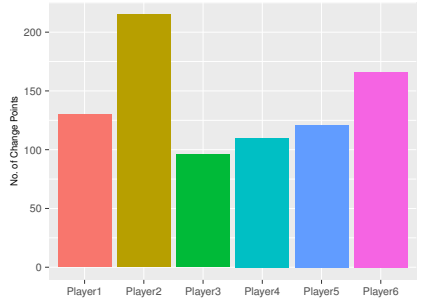

Fig. 6: Number of detected change points with respect to each player for one session

\section{Analysis \& Player Ratings}

After dividing the data set into frames using detected change points, we analyze different player attributes. In each frame we calculate the dominant class label and assign that class label to the whole frame. Then we calculate the number of passes by counting the number of frames labeled as pass. We do this accordingly for kick, sprint and dribbling events. We also calculate the total distance covered by the player. We compare our performance attributes with Zepp sensor in Table II. The first term in each of the attribute column represents Zepp sensor value and the second term is the value calculated using our model. The third column states in which position the player was playing. We can see that in most of the cases the calculated metrics are close. SoccerMate detected more kicks than Zepp model. There is a quite deviation in the total distance calculation and we would like to further investigate this discrepancy in future. As Zepp did not provide any information on dribbling event so we did not include the number of dribbling in Table II. After calculating the metrics, we calculate the ratings of each player using our scoring model. In figure 7 calculated individual ratings of each player is shown. 


\section{CONCLUSION}

In this paper we demonstrated a personal soccer attribute profiling model - SoccerMate that helps users to build their soccer skills without any professional settings. We extract and infer general soccer events using the accelerometer data of regular wearable devices. We also exploit deep learning method in our pipeline to build a scalable recognition model. After extracting soccer event information, we analyze and derive performance analytics of the user. We also described a rating model based on attribute coefficients of FIFA 2013 video game. We validate our model using real life implementation and describe the resource usages of our model. Using SoccerMate we only profile the general attributes of the user but for proper profiling we need to consider more constraints. Position specific attributes provide more meaningful insights about the performance of a player. We intend to augment more sensor modalities in future to perform more fine grained profiling of soccer metrics.

\section{REFERENCES}

[1] "Sensoria," http://www.sensoriafitness.com.

[2] "Catapult sports," http://www.catapultsports.com.

[3] "Motus baseball," http://www.motusglobal.com/motusbaseball.html.

[4] “Zephyr," https://www.zephyranywhere.com/system/components.

[5] "germany2014," https://www.wareable.com/sport/ the-world-cup-wearable.

[6] M. Bächlin, K. Förster, and G. Tröster, "Swimmaster: a wearable assistant for swimmer," in UbiComp 2009: Ubiquitous Computing, 11th International Conference, UbiComp 2009, Orlando, Florida, USA, September 30 - October 3, 2009, Proceedings, 2009, pp. 215-224.

[7] L. N. N. Nguyen and D. e. a. Rodríguez-Martín, "Basketball activity recognition using wearable inertial measurement units," in Proceedings of the XVI International Conference on Human Computer Interaction, 2015, pp. 60:1-60:6.

[8] R. Shah and R. Romijnders, "Applying deep learning to basketball trajectories," CoRR, vol. abs/1608.03793, 2016. [Online]. Available: http://arxiv.org/abs/1608.03793

[9] B. Zhou, H. Koerger, M. Wirth, C. Zwick, C. Martindale, H. Cruz, B. Eskofier, and P. Lukowicz, "Smart soccer shoe: monitoring football interaction with shoe integrated textile pressure sensor matrix," in Proceedings of the 2016 ACM International Symposium on Wearable Computers, ISWC 2016, Heidelberg, Germany, September 12-16, 2016, 2016, pp. 64-71.

[10] J. Lloret, M. Garcia, A. Catala, and J. J. P. C. Rodrigues, "A groupbased wireless body sensors network using energy harvesting for soccer team monitoring," IJSNet, vol. 21, no. 4, pp. 208-225, 2016.

[11] R. Afrouzian, H. Seyedarabi, and S. Kasaei, "Pose estimation of soccer players using multiple uncalibrated cameras," Multimedia Tools Appl., vol. 75, no. 12, pp. 6809-6827, 2016.

[12] S. Baysal and P. Duygulu, "Sentioscope: A soccer player tracking system using model field particles," IEEE Trans. Circuits Syst. Video Techn., vol. 26, no. 7, pp. 1350-1362, 2016.

[13] D. Schuldhaus, C. Jakob, C. Zwick, H. Koerger, and B. M. Eskofier, "Your personal movie producer: generating highlight videos in soccer using wearables," in Proceedings of the 2016 ACM International Symposium on Wearable Computers, ISWC 2016, Heidelberg, Germany, September 12-16, 2016, 2016, pp. 80-83.

[14] "engsoccerdata," https://github.com/jalapic/engsoccerdata.

[15] "Footballdata," https://github.com/jokecamp/FootballData.

[16] “openfootball," https://github.com/openfootball.

[17] “soccer," https://www.kaggle.com/hugomathien/soccer.

[18] "Soccerdb," https://datahub.io/dataset?tags=soccer.

[19] L. Gyarmati and R. Stanojevic, "Qpass: a merit-based evaluation of soccer passes," CoRR, vol. abs/1608.03532, 2016. [Online]. Available: http://arxiv.org/abs/1608.03532

[20] L. Cotta and P. O. V. de Melo et al, "Using fifa soccer video game data for soccer analytics," Workshop on Large Scale Sports Analytics, 2016.
[21] Y. Saito, M. Kimura, and S. Ishizaki, "Real-time prediction to support decision-making in soccer," in KDIR 2015 - Proceedings of the International Conference on Knowledge Discovery and Information Retrieval, 2015, pp. 218-225.

[22] J. Brooks, M. Kerr, and J. Guttag, "Developing a data-driven player ranking in soccer using predictive model weights," in Proceedings of the 22Nd ACM SIGKDD International Conference on Knowledge Discovery and Data Mining, ser. KDD '16, 2016, pp. 49-55.

[23] G. J. Szekely and M. L. Rizzo, "Hierarchical clustering via joint between-within distances: Extending ward's minimum variance method," Journal of Classification, vol. 22, no. 2, pp. 151-183, 2005.

[24] M. L. Rizzo and G. J. Székely, "DISCO analysis: A nonparametric extension of analysis of variance," 2010.

[25] D. S. Matteson and N. A. James, "A Nonparametric Approach for Multiple Change Point Analysis of Multivariate Data," Journal of the American Statistical Association, 2014.

[26] “Zepp soccer," http://www.zepp.com/en-us/soccer/. 